\title{
UKnowledge
}

University of Kentucky

UKnowledge

\section{Intrathecal Administration of AYX2 DNA Decoy Produces a Long- Term Pain Treatment in Rat Models of Chronic Pain by Inhibiting the KLF6, KLF9, and KLF15 Transcription Factors}

Julien Mamet

Adynxx, Inc.

Michael Klukinov

Stanford University

Scott Harris

Adynxx, Inc.

Donald C. Manning

Adynxx, Inc.

Simon Xie

Afll $\$ W_{i}$ thing and additional works at: https://uknowledge.uky.edu/physiology_facpub

Part of the Analytical, Diagnostic and Therapeutic Techniques and Equipment Commons,

Pharmacology Commons, Pharmacy and Pharmaceutical Sciences Commons, and the Physiology

See next page for additional authors

Commons

Right click to open a feedback form in a new tab to let us know how this document benefits you.

\section{Repository Citation}

Mamet, Julien; Klukinov, Michael; Harris, Scott; Manning, Donald C.; Xie, Simon; Pascual, Conrado; Taylor, Bradley K.; Donahue, Renee R.; and Yeomans, David C., "Intrathecal Administration of AYX2 DNA Decoy Produces a Long-Term Pain Treatment in Rat Models of Chronic Pain by Inhibiting the KLF6, KLF9, and KLF15 Transcription Factors" (2017). Physiology Faculty Publications. 104.

https://uknowledge.uky.edu/physiology_facpub/104

This Article is brought to you for free and open access by the Physiology at UKnowledge. It has been accepted for inclusion in Physiology Faculty Publications by an authorized administrator of UKnowledge. For more information, please contact UKnowledge@lsv.uky.edu. 


\title{
Intrathecal Administration of AYX2 DNA Decoy Produces a Long-Term Pain Treatment in Rat Models of Chronic Pain by Inhibiting the KLF6, KLF9, and KLF15 Transcription Factors
}

\author{
Digital Object Identifier (DOI) \\ https://doi.org/10.1177/1744806917727917 \\ Notes/Citation Information \\ Published in Molecular Pain, v. 13, p. 1-17. \\ (C) The Author(s) 2017
}

This article is distributed under the terms of the Creative Commons Attribution-NonCommercial 4.0 License (http://www.creativecommons.org/licenses/by-nc/4.0/) which permits non-commercial use, reproduction and distribution of the work without further permission provided the original work is attributed as specified on the SAGE and Open Access pages (https://us.sagepub.com/en-us/nam/openaccess-at-sage).

\section{Authors}

Julien Mamet, Michael Klukinov, Scott Harris, Donald C. Manning, Simon Xie, Conrado Pascual, Bradley K. Taylor, Renee R. Donahue, and David C. Yeomans 


\author{
Julien Mamet', Michael Klukinov², Scott Harris', Donald \\ C Manning', Simon Xie ${ }^{3}$, Conrado Pascual ${ }^{3}$, Bradley K Taylor ${ }^{4}$, \\ Renee R Donahue ${ }^{4}$ and David C Yeomans ${ }^{2}$
}

\begin{abstract}
Background: Nociception is maintained by genome-wide regulation of transcription in the dorsal root ganglia-spinal cord network. Hence, transcription factors constitute a promising class of targets for breakthrough pharmacological interventions to treat chronic pain. DNA decoys are oligonucleotides and specific inhibitors of transcription factor activities. A methodological series of in vivo-in vitro screening cycles was performed with decoy/transcription factor couples to identify targets capable of producing a robust and long-lasting inhibition of established chronic pain. Decoys were injected intrathecally and their efficacy was tested in the spared nerve injury and chronic constriction injury models of chronic pain in rats using repetitive von Frey testing.

Results: Results demonstrated that a one-time administration of decoys binding to the Kruppel-like transcription factors (KLFs) 6, 9, and 15 produces a significant and weeks-month long reduction in mechanical hypersensitivity compared to controls. In the spared nerve injury model, decoy efficacy was correlated to its capacity to bind KLFI5 and KLF9 at a specific ratio, while in the chronic constriction injury model, efficacy was correlated to the combined binding capacity to KLF6 and KLF9. AYX2, an 18-bp DNA decoy binding KLF6, KLF9, and KLFI5, was optimized for clinical development, and it demonstrated significant efficacy in these models.

Conclusions: These data highlight KLF6, KLF9, and KLFI 5 as transcription factors required for the maintenance of chronic pain and illustrate the potential therapeutic benefits of AYX2 for the treatment of chronic pain.
\end{abstract}

\author{
Keywords \\ Chronic pain, Kruppel-like factors, KLF6, KLF9, KLFI5, DNA decoy, AYX2
}

Date received: 17 May 2017; accepted: 27 July 2017

\title{
Introduction
}

Chronic pain stems, in part, from an acquired and maintained pathologic sensitization of the dorsal root ganglion (DRG) - spinal cord network. Microarray experiments revealed that sensitization is maintained by sequential waves of gene regulation. ${ }^{1-3}$ Those can impact up to $\sim 400$ genes at a time across a broad spectrum of gene categories, including genes controlling the transcriptional, homeostatic, metabolic, oxidative, protein turnover, trafficking, structural, and communicative

\footnotetext{
'Adynxx, Inc., CA, USA

${ }^{2}$ Faculty of Anesthesia, Stanford University, CA, USA

${ }^{3} \mathrm{AfaSci}$, Inc, CA, USA

${ }^{4}$ Department of Physiology, University of Kentucky, KY, USA
}

Corresponding author:

Julien Mamet, Adynxx, Inc, 100 Pine St., Suite 500, San Francisco,

CA 94III, USA.

Email: jmamet@adynxx.com 
states of neurons and glia. ${ }^{1,4-15}$ Dynamically, these expression changes are launched early on by immediate-early transcription factors like early growth factor 1 (EGR1) following an injury and maintained over time by delayed transcription factor networks. ${ }^{16-18}$

Transcription factors directly regulate gene expression, with a single factor regulating the expression of many genes. As such, they represent therapeutic targets with the unique opportunity to simultaneously influence the activity of a high number of genes supporting nociception and influence the long-term course of pain. From a therapeutic prospective, transcription factors can be inhibited by DNA decoys or synthetic double-stranded oligonucleotides that mimic their binding sites on the genome (Figure 1). In a prior study, we demonstrated that locally blocking EGR1 activity with an EGR1specific decoy named AYX1 around the time of an injury prevents the development of long-term pain in rats. ${ }^{18}$ AYX1 is currently under clinical development (clinicaltrial.gov identifier: NCT02807428).

We hypothesized that a brief inhibition of select delayed transcription factors maintaining the stability of transcriptomes associated with pathologic nociception could reduce pain for long periods of time. An underlying assumption was that once those transcriptomes are destabilized, they are unlikely to spontaneously reinitiate because their occurrence depends on earlier transcriptional waves that have resolved in mature pain states. To test this hypothesis, we designed 35 decoys against single factors or family of factors with the potential to be involved in the maintenance of pathologic nociception. A screening method was implemented to test the decoys in vivo for their capacity to reduce mechanical hypersensitivity in the spared nerve injury (SNI) model of chronic neuropathic pain. The SNI model was chosen for its well-characterized neuropathic pain features and prior
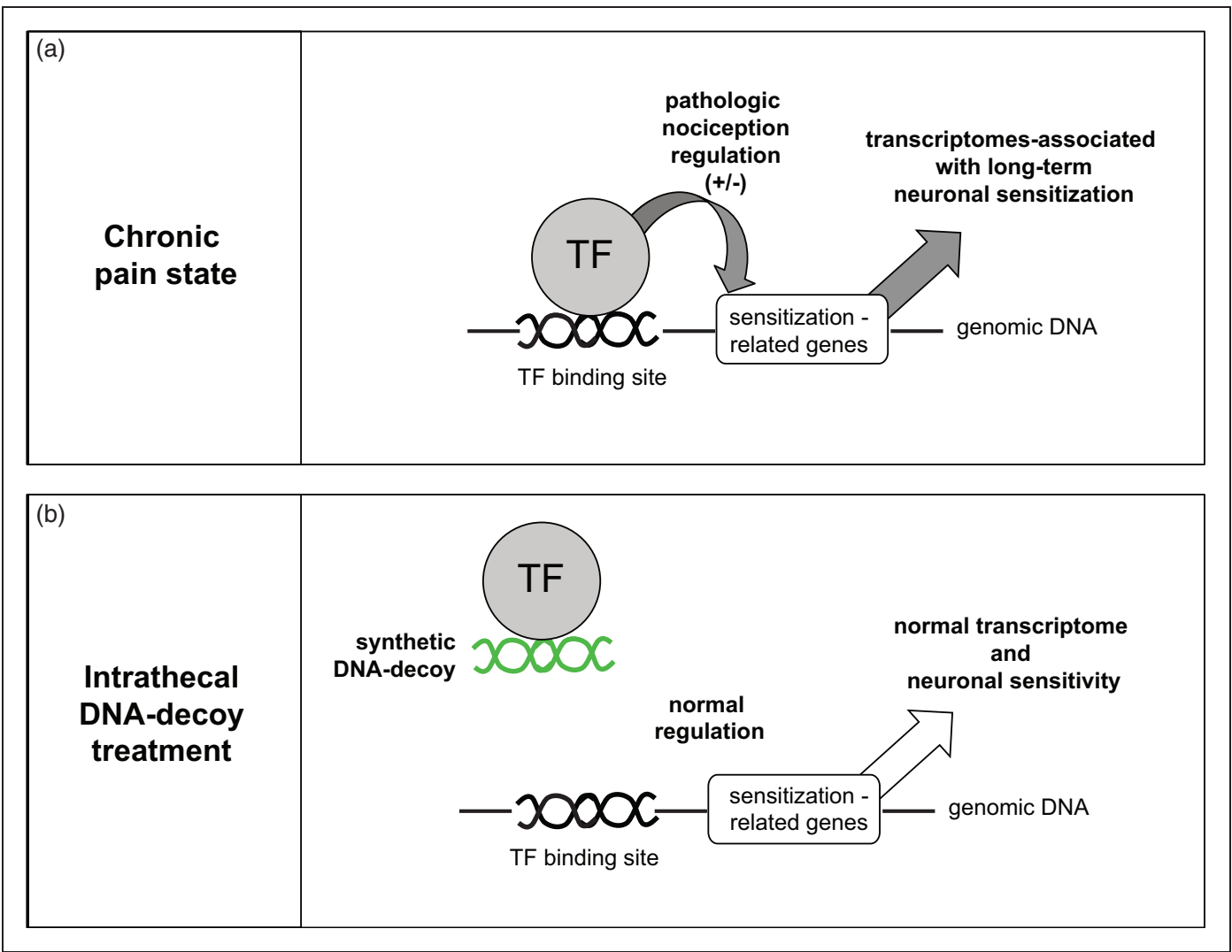

Figure I. Illustration of DNA decoy action in chronic pain treatment. In a chronic pain state (a), TFs involved in the maintenance of neuronal sensitization bind to specific DNA sequences (TF binding site) on the genome of DRG and spinal cord cells and upregulate or downregulate (pathologic nociception regulation) hundreds of genes (sensitization-related genes). As a result, the genomic status of those cells is shifted toward a pathologic set of gene regulations that are responsible for the maintenance of neuronal sensitization and chronic pain (transcriptomes associated with long-term neuronal sensitization). DNA decoys (green) are synthetic, double-stranded oligonucleotides that mimic the binding sites of their TF target on the genome. The local intrathecal administration of specific DNA decoys (b) can prevent the binding of TF to their binding sites on the genome and interrupt the maintenance of the transcriptomes associated with long-term neuronal sensitization, resetting gene regulation toward a normal transcriptome and a normal neuronal sensitivity. TF: transcription factor. 
demonstration with AYX1 that it can be sensitive to an intrathecal decoy treatment. ${ }^{18}$ In the light of a high number of decoys to test, screening throughput and animal use considerations, the decoy attrition strategy relied on initially detecting potential efficacy in small groups of animals and assessing accuracy and reproducibility of those effects by their independent reproduction through multiple screening phases. Ultimately, efficacy reproduced through the screening was confirmed in larger groups of animals. Out of the screen, only two decoys produced a significant and long-term reduction in mechanical hypersensitivity, both targeting the Kruppel-like factors (KLFs) family. Iterative in vivoin vitro testing cycles identified the specific KLF factors and decoy binding behaviors associated with each decoy's activity. AYX2 was developed using this knowledge as a drug candidate with the potential to provide long-term reduction of chronic pain in patients with a single administration.

\section{Methods}

\section{Decoys and vehicles}

Decoys were designed at Adynxx, except for KLF reference decoys 1 and 2, which were selected from the literature (supplemental Table 1). ${ }^{19,20}$ Sense and antisense strands of each decoy were manufactured by Invitrogen (CA), resuspended in Tris- and calcium-based vehicle formulations, and hybridized at room temperature.

\section{Enzyme-linked immunosorbent assay}

Decoy binding to KLFs was measured using a customized version of a commercial Enzyme-linked immunosorbent assay (ELISA) kit (Affymetrix, CA). Briefly, 12.8 pmoles of KLF biotin-decoy probe were incubated with $15 \mu \mathrm{g}$ of HELA nuclear protein extracts for KLF1-6, KLF8-14, and KLF16-17 detection (catalogue \# 36010, Active motif, CA) and HEK290 extracts for KLF15 detection (catalogue \# 36033, Active motif, CA). For KLF7 detection, 0.5 and $1 \mu \mathrm{g}$ of a recombinant human KLF7 protein was utilized (Novus, CO). The probe-protein mix was processed following the kit's instructions and the quantity of captured KLF measured with an antibody-based colorimetric detection in a microplate reader $\left(\mathrm{OD}_{450} \mathrm{~nm}\right)$. Primary antibodies were commercial antibodies validated for specificity by suppliers and characterized for ELISA or assays detecting the native, human form of their target (e.g., gel shift). They are listed in supplemental Table 2. The secondary antibody conjugated to horseradish peroxidase was provided in the ELISA kit (dilution 1:200). The experimental conditions for assay linearity, specificity, and sensitivity were determined using KLF decoy 1, the most efficacious KLF decoy identified during the initial in vivo screening, to the human form of KLF4, a predominant KLF member known to bind the KLF consensus binding sites that were used as a frame to design $K L F$ decoy 1 in silico. Assay sensitivity was optimized by testing the binding level of a fixed amount of $K L F$ decoy 1 biotinylated probe incubated in the presence of crossover quantities of nuclear protein extracts and KLF4 primary antibody dilutions. Assay specificity was confirmed by standard controls previously described for transcription factor ELISA $^{18,21}$ : (1) nonspecific, background noise correction using a reaction mix lacking proteins internally for each individual assay and testing condition, (2) binding competition experiments to the $K L F$ decoy $l$ biotin probe with free, nonbiotinylated $K L F$ decoy 1 competitor, (3) confirmation of binding by positive control decoys $K L F$ reference decoys 1 and 2, and (4) confirmation of absence of binding by two complementary mutant, non-KLF binding decoys. The expansion of the assay beyond KLF4 to the other KLFs was performed using the same amount of nuclear proteins extract (except for KFL7, see above) and the primary antibody dilution recommended by the supplier when available or the lowest practical dilution with specificity measured against the binding to a mutant decoy.

\section{Animals}

A total of 229 Sprague Dawley rats (Harlan Industries, Livermore, CA), males, 250-300 g, were used. Animal housing and handling was performed in accordance with the Animal Care and Use Committees guidelines of each testing site. Those committees approved all experiments.

\section{Vehicle and decoy administration}

Articles were delivered via a bolus, percutaneous $20 \mu \mathrm{L}$ intrathecal injection at the L5-L6 vertebra level as previously described. ${ }^{18}$ Throughout the study, decoys were dosed between 50 and 300 nmoles, which is consistent with prior work with AYX1 decoy showing that those doses injected intrathecally in rats are sufficient to produce a pharmacological effect. ${ }^{18}$

\section{Behavioral testing}

SNI and chronic constriction injury (CCI) models were performed as described elsewhere. ${ }^{22-24}$ Rats were habituated in cages with mesh wire floors for $1 \mathrm{~h}$ before von Frey hairs testing. Calibrated von Frey hairs were applied with the following pseudorandom pattern: 6,1 , 10, 4, 26, 10, and $8 \mathrm{~g}$. For each testing level, von Frey hairs were applied five times consecutively to the hind paw ipsilateral to the injury, and the number of paw 
withdrawals ( 0 to 5 per level, 0 to 35 in total) was recorded as a response. The time interval between each testing level for a given rat was at least 3 min. ${ }^{18}$

\section{Blinding and randomization}

Behavioral experiments were all performed blinded. Each testing site received blinded article vials, and the blinding code was revealed to the testing site and experimenter only after testing was completed and the data received. Data were analyzed independently at Adynxx and at the testing sites, and the analyses were crosschecked. Animal randomization to treatment groups was performed before treatment on the day of the first dosing: animals were distributed in groups so that the mean von Frey responses were close across testing groups, targeting within $15 \%$ of each other if values permitted.

\section{Predetermined inclusion and exclusion criteria}

Due to the amount of testing required for this work, appropriate animal model performance and technical bias avoidance were pursued by predetermining the following inclusion/exclusion criteria derived from prior experimental work ${ }^{18,25}$ instead of using sham groups: (1) threshold for the absence/presence of model-induced hypersensitivity: animals with von Frey values $\leqslant 5$ on the day of the first dosing were excluded since rats can reach this response level in basal conditions and (2) pain model performance: if the average von Frey values of vehicletreated rats decreased by $50 \%$ or more during the first week following injection, then the entire tested cohort (vehicle- plus decoy-treated animals) was excluded. When they occurred, exclusions are listed in the figure legends, and the number of animals listed per testing conditions reflects the exclusions.

\section{Statistical analysis}

Nonparametric Student's $t$ test was used to analyze individual conditions and whole data distribution comparison between experimental conditions using Microsoft Excel, version 14.4.1. One-way analysis of variance and Dunnett's multiple comparison tests were performed using GraphPad Prism version 7.0 (GraphPad Software, La Jolla, CA, www.graphpad.com).

\section{Results}

\section{Decoys efficacy screening in the SNI model}

Thirty-five transcription factors or families of transcription factor targets were selected for screening based on their known function in pain or pain-related biological systems, such as inflammation or memory and internal cross-species analysis of selected pain-related gene promoters (Table 1).

Decoys targeting those factors were designed in silico using response element matrices and binding prediction software (e.g., TESS, TRANSFAC, JASPAR). Decoys were grouped in 11 triplexes and one duplex based on the functionality of their target to increase testing throughput (TLX, Table 1). The screening was structured in three sequential phases of attrition: (1) phase 1: identify triplex/duplex with potential efficacy for reducing mechanical hypersensitivity in the SNI model, (2) phase 2: independent confirmation of their efficacy, and (3) phase 3: identification of the individual decoys that carry efficacy. In each phase, articles were injected once at day 14 (15 in one occurrence) following SNI surgery, at which point mechanical hypersensitivity was developed, and once more within 10 days to increase the reliability of tested outcomes. Due to the high number of decoys to test, phase 1 was performed with $n=3$ for each triplex/duplex, and the accuracy of effects was pursued via their independent reproduction throughout the screening. This strategy was supported by prior work with the AYX1 decoy showing that meaningful decoydriven pharmacodynamic effects can be detected with $n=3$ in the SNI model. ${ }^{18}$ The triplex/duplex approach introduced the possibility of interactions between the effects of decoys when injected together that could mask the effects decoys could have individually. Therefore, the choice was made in phase 2 , while " $n$ " increased to 5-6 to only seek for trends of efficacy over using a strict statistical analysis to guide triplex selection. In that context, the criteria to define trends of efficacy in phases 1 and 2 were (1) total von Frey hair responses lower compared to vehicle-treated animals and (2) a numerical reduction of at least $25 \%$ from the baseline hypersensitivity measured on day 14 for at least two consecutive time points. Statistical analysis was then performed for individual decoys testing in phase 3 . Overall, data normalization was an essential tool of the decoy screening and optimization process that allowed for a sensitive analysis of data across small groups of animals, independent testing and laboratories. The corresponding raw von Frey data are presented in supplemental tables.

In the first phase of the screening, only the effects of TLX1, TLX4, and TLX5 matched our dual selection criteria for efficacy, and their effect was tested for approximately three weeks (Figure 2(a)).

TLX7 and TLX8 showed some potential for efficacy early on, so they were also followed over three weeks, but their effects did not meet our dual criteria at the end (Figure 2(a) and (b)). No trend of effect was suggested by TLX6, TLX9, TLX10, or TLX11 treatments, which illustrates the specificity of the triplex/duplex approach 
Table I. Decoy triplexes and transcription factor targets.

\begin{tabular}{|c|c|c|c|c|c|c|}
\hline \multirow{2}{*}{$\begin{array}{l}\text { Triplex } \\
\text { number }\end{array}$} & \multirow{2}{*}{$\begin{array}{l}\text { Screening } \\
\text { decoy } \\
\text { number }\end{array}$} & \multicolumn{5}{|c|}{ Transcription factor target } \\
\hline & & Functional scope & Name & Superclass & Class & Family \\
\hline & Decoy 2 & & EGRI & Zinc finger & $\mathrm{Zn}$ finger - $\mathrm{C} 2 \mathrm{H} 2$ & $\begin{array}{c}\text { Developmental/cell } \\
\text { cycle regulator }\end{array}$ \\
\hline \multirow[t]{3}{*}{ TLXI } & Decoy 4 & $\begin{array}{l}\text { Immediate early } \\
\text { gene }\end{array}$ & CEBP & Basic domains & bZIP & CAAT enhancer \\
\hline & Decoy 31 & & TCF/SRF & Beta scaffold & MADs box & $\begin{array}{l}\text { Responders to } \\
\text { external signals }\end{array}$ \\
\hline & Decoy 5 & & CREB/ATF & Basic domains & bZIP & CREB \\
\hline \multirow[t]{3}{*}{ TLX2 } & Decoy I & Constitutive factor & $\mathrm{API}$ & Basic domains & bZIP & API \\
\hline & Decoy 9 & & ETSI/SRF/ElkI & $\begin{array}{l}\text { Helix-turn-helix/ } \\
\text { beta scaffold }\end{array}$ & $\begin{array}{l}\text { Tryptophan } \\
\text { cluster/MADS }\end{array}$ & $\begin{array}{l}\text { ETS/responder to } \\
\text { external signals }\end{array}$ \\
\hline & Decoy 26 & & REST & Zinc finger & $\mathrm{Zn}$ finger $-\mathrm{C} 2 \mathrm{H} 2$ & $\begin{array}{c}\text { Developmental/cell } \\
\text { cycle regulators }\end{array}$ \\
\hline \multirow[t]{3}{*}{ TLX3 } & Decoy 27 & $\begin{array}{l}\text { Tissue/neuro } \\
\text { differentiation }\end{array}$ & RUNX & Beta scaffold & Runt & Runt \\
\hline & Decoy 12 & & HNFI & Helix-turn-helix & Homeo domain & Homeo domain \\
\hline & Decoy 6 & & ELfI/POUIFI & Helix-turn-helix & Tryptophan cluster & ETS \\
\hline \multirow[t]{3}{*}{ TLX4 } & Decoy 7 & $\begin{array}{l}\text { Tissue/neuro } \\
\text { differentiation }\end{array}$ & ELKI & Helix-turn-helix & Tryptophan cluster & ETS \\
\hline & Decoy 8 & & ELKI/ETSI & Helix-turn-helix & Tryptophan cluster & ETS \\
\hline & Decoy 16 & & KLFs & Zinc finger & $\mathrm{Zn}$ finger $-\mathrm{C} 2 \mathrm{H} 2$ & KLF Like \\
\hline \multirow[t]{3}{*}{ TLX5 } & Decoy 17 & $\begin{array}{l}\text { Tissue/neuro } \\
\text { differentiation }\end{array}$ & KLFs & Zinc finger & Zn finger - $\mathrm{C} 2 \mathrm{H} 2$ & KLF Like \\
\hline & Decoy 35 & & LEFI & Beta scaffold & HMG & TCF-I \\
\hline & Decoy 18 & & MAF & Basic domain & bZIP & Maf \\
\hline \multirow[t]{3}{*}{ TLX6 } & Decoy 19 & $\begin{array}{c}\text { Development/cell } \\
\text { differentiation }\end{array}$ & MEF2 & Beta scaffold & MADs box & $\begin{array}{l}\text { Regulator of } \\
\text { differentiation }\end{array}$ \\
\hline & Decoy 22 & & Ngfma & N/A & N/A & N/A \\
\hline & Decoy 23 & & $\mathrm{POU} 2 \mathrm{FI} / 3 \mathrm{~F} / 5 \mathrm{~F}$ & Helix-turn-helix & Homeo domain & $\begin{array}{l}\text { POU domain } \\
\text { factors }\end{array}$ \\
\hline \multirow[t]{3}{*}{ TLX7 } & Decoy 24 & $\begin{array}{c}\text { Development/cell } \\
\text { differentiation }\end{array}$ & POU4FI & Helix-turn-helix & Homeo domain & $\begin{array}{l}\text { POU domain } \\
\text { factors }\end{array}$ \\
\hline & Decoy II & & GATAI & Zinc finger & Zn finger - Cys 4 & GATA \\
\hline & Decoy 28 & & SPI & Zinc finger & $\mathrm{Zn}$ finger $-\mathrm{C} 2 \mathrm{H} 2$ & Ubiquitous Factors \\
\hline \multirow[t]{2}{*}{ TLX8 } & Decoy 33 & $\begin{array}{l}\text { Ubiquitous/broad } \\
\text { scope }\end{array}$ & USF & Basic domains & bHLH-ZIP & $\begin{array}{l}\text { Ubiquitous bHLH- } \\
\text { ZIP factors }\end{array}$ \\
\hline & Decoy 3 & & $\begin{array}{l}\text { CACCC-box } \\
\text { binding factors }\end{array}$ & N/A & N/A & N/A \\
\hline
\end{tabular}


Table I. Continued

\begin{tabular}{|c|c|c|c|c|c|c|}
\hline \multirow{2}{*}{$\begin{array}{l}\text { Triplex } \\
\text { number }\end{array}$} & \multirow{2}{*}{$\begin{array}{l}\text { Screening } \\
\text { decoy } \\
\text { number }\end{array}$} & \multicolumn{5}{|c|}{ Transcription factor target } \\
\hline & & Functional scope & Name & Superclass & Class & Family \\
\hline \multirow[t]{3}{*}{ TLX9 } & Decoy 30 & $\begin{array}{r}\text { Transcription } \\
\text { machinery }\end{array}$ & TBP & Beta-scaffold & $\begin{array}{l}\text { TATA-binding } \\
\text { proteins }\end{array}$ & TBP \\
\hline & Decoy 21 & & NFY & Beta-scaffold & HMG & NFY \\
\hline & Decoy 34 & & ZFHX3 & Helix-turn-helix & Homeo domain & $\begin{array}{l}\text { Homeo domain } \\
\text { plus zinc finger } \\
\text { motifs }\end{array}$ \\
\hline \multirow[t]{3}{*}{ TLXIO } & Decoy 20 & $\mathrm{Ca}^{++}$sensitive & NFAT & Beta scaffold & RHR & NFAT \\
\hline & Decoy 15 & & KCNIP3 & E2F hand & $N / A$ & $N / A$ \\
\hline & Decoy 14 & & IRF & Homeo domain & Tryptophan cluster & $\begin{array}{l}\text { Interferon-regulat- } \\
\text { ing factors }\end{array}$ \\
\hline \multirow[t]{3}{*}{ TLXII } & Decoy 25 & $\begin{array}{l}\text { External signal } \\
\text { response }\end{array}$ & PPAR alpha & Zinc finger & Zn finger - Cys 4 & $\begin{array}{l}\text { Thyroid hormone } \\
\text { receptor-like } \\
\text { factors }\end{array}$ \\
\hline & Decoy 13 & & HSF & Helix-turn-helix & Heat shock & HSF \\
\hline & Decoy 29 & Mixed & STAT & Beta scaffold & STAT & STAT \\
\hline \multirow[t]{2}{*}{ TLX 12} & Decoy 10 & & ETSI & Helix-turn-helix & Tryptophan cluster & ETS \\
\hline & Decoy 32 & & TEADI & Basic domain & bZIP & bZIP-PAR \\
\hline
\end{tabular}

bZIP: basic leucine zipper; bHLH-ZIP: helix-loop-helix/leucine zipper factors; Screening decoy 16: KLF decoy I; Screening decoy I7: KLF decoy 2; Screening decoy 3: CACCC-box decoy; neuro: neurological tissue; N/A: not applicable.

(Figure 2(b)). Finally, the effects of TLX2, TLX3 and TLX12 suggested a potential aggravation of mechanical hypersensitivity (Figure 2(b)). In the second phase, TLX1 and TLX5 but not TLX4 reproduced efficacy by meeting our dual criteria (Figure 3(a)). TLX8, which only met the efficacy criteria against vehicle in phase 1 , was also tested here to further assess for the sensitivity of our approach. Consistent with phase 1, it met only one predefined criteria for efficacy but not the two (Figure 3(a)).

TLX1 and TLX5 were then carried into the final phase of the screening to evaluate the efficacy of their respective individual decoys. Two hundred nanomoles of each decoy were injected versus 100 nmoles in their respective triplex (i.e., maximal dose due to solubility and dosing volume limits as each triplex contained three different molecular entities). Individual decoys from TLX1 only demonstrated a modest efficacy that was short-lived and that could not be amplified by a second injection (Figure 3(b)). From TLX5, the two decoys binding KLF factors, KLF decoys 1 and 2, produced a robust and long-lasting reduction in mechanical hypersensitivity that was maintained numerically over controls until the hypersensitivity from the model started to resolve $\sim 5$ weeks after injections (Figure 3(c)). In contrast, the LEF1 decoy 35 showed a limited efficacy that was numerically lower than the triplex efficacy (Figure 3(c)). Interestingly, TLX8, which showed potential efficacy during the screening, contains a CACCC-box decoy, which has a consensus site for the $\mathrm{SP} / \mathrm{KLF}$ family different from $K L F$ decoy 1 and 2. These results suggested differential KLF binding patterns for the KLF decoys and raised the question of which KLFs are involved in chronic pain. Raw von Frey data for each group and testing phase of the screening are presented in supplemental Table 3.

\section{Decoy KLF binding patterns}

The KLF family includes 17 members, each recognizing a range of DNA sequences. ${ }^{26,27} K L F$ decoys 1 and 2 were designed in silico. To decipher their actual KLF binding pattern, a KLF ELISA assay was developed to measure their binding activity against the human forms of the 17 family members (Figure 4(a)-(c)). Binding activities of $K L F$ decoys 1 and 2 were compared to (1) the CACCC-box decoy from TLX8, (2) two independent $K L F$ reference decoys containing complementary versions of the KLF consensus-binding site selected from the literature, ${ }^{19,20}$ and (3) to negative control-mutant 


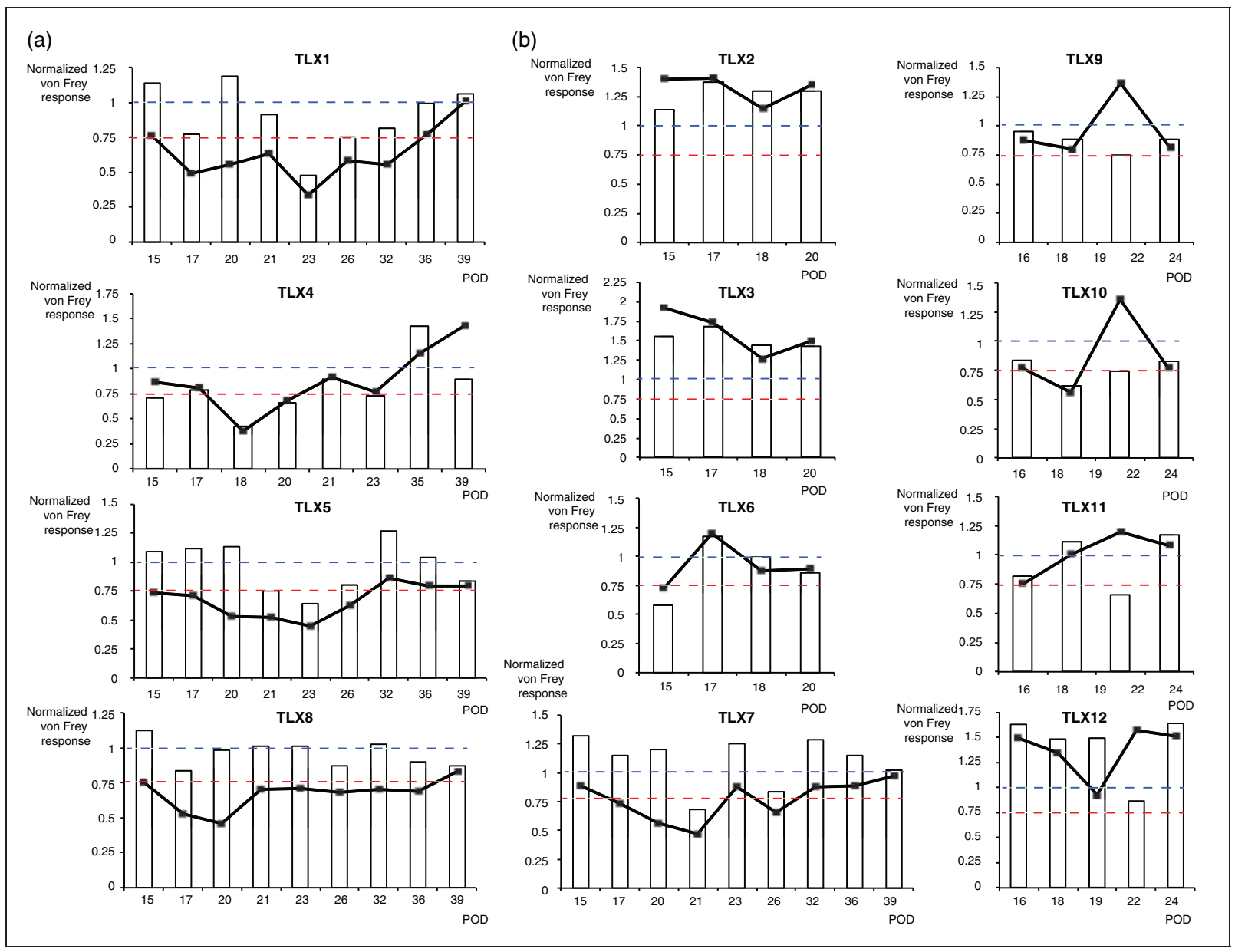

Figure 2. Effects of the triplex/duplex in the phase I of the decoy screening in the SNI model. Decoys' performance relating to the dual efficacy criteria are shown: von Frey values normed on POD 14 values before the first injection (white bars), and von Frey values normed on vehicle values at each tested day (black curves). Blue dashed lines mark the normed threshold value of I to assess decoys performance (black curves) against vehicle. Red dashed lines mark the normed threshold value of 0.75 (i.e., $25 \%$ reduction) to assess decoys performance (white bars) against PODI4 preinjection von Frey values. von Frey values were normalized individually for each rat, and data are presented as means for each group; corresponding raw von Frey values are presented in supplemental Table 3. The triplexes meeting the predefined criteria of potential efficacy are shown in (a) while the ones that did not are shown in (b). Each triplex contained I00 nmoles of each decoy and the duplex TLXI2 contained I50 nmoles of each decoy. Dosing occurred once at day I4 (day I5 for TLXI0) and day I7 (day 18 for TLXI0); $n=3$ for each triplex/duplex and $n=4$ for vehicle. This study was performed at the Stanford University. POD: post-surgery day.

decoys. ${ }^{18}$ Positive binding was found for at least one decoy to KLF4, KLF5, KLF6, KLF7, KLF9, KLF12, KLF15, and KLF16 (Table 2). No relevant binding was detected for the other KLFs under our testing conditions. Importantly, decoys all presented different KLF binding patterns (Figure 4(d) and Table 2), consistent with the different efficacy profiles observed for the KLF decoys in vivo.

\section{KLF decoys with combined binding to KLF6, KLF9, and KLFI5 treat chronic pain}

To identify which KLFs are associated to a decoy's efficacy for reducing pain and if they differ with the type of injury, $K L F$ decoy 1 and 2 were tested in parallel to the $C A C C C$-box decoy and to the two KLF reference decoys in the SNI (i.e., nerve transection) and CCI models (i.e., nerve ligature). Two hundred nanomoles of each decoy were injected at day 14 and 17 , similar to the phase 3 of the screen. In the SNI model, KLF decoy 1 and $K L F$ reference 1 both produced a maximum mechanical hypersensitivity reduction of $\sim 70 \%$, and their effect was observed until hypersensitivity was resolving in controls (Figure 4(e) and supplemental Table 4). $K L F$ decoy 2 produced efficacy in a similar range in terms of amplitude and duration, although numerically lower. The CACCC-box decoy produced an intermediate level of efficacy that resolved earlier; therefore, its testing was 


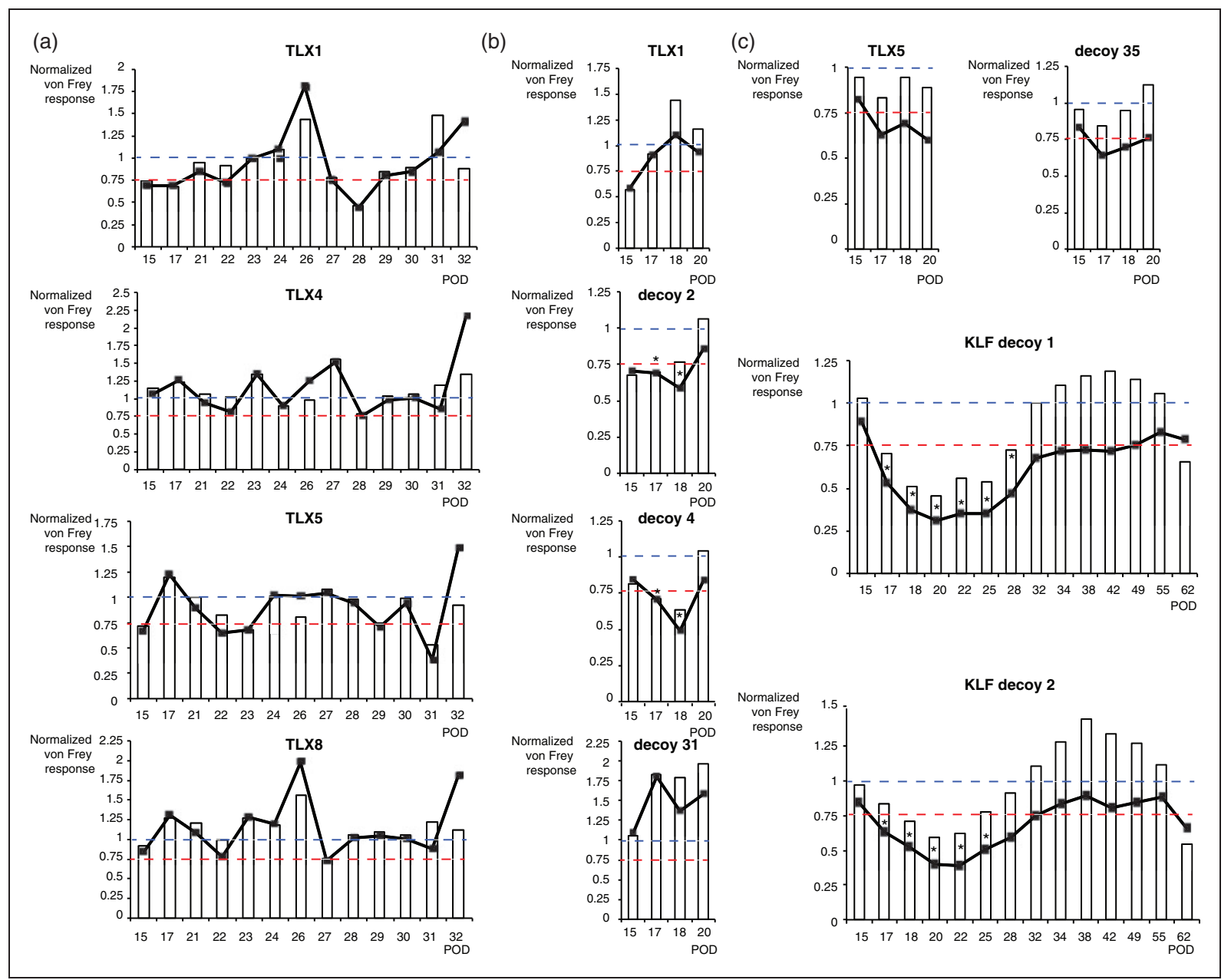

Figure 3. (a) Effects of TLXI, TLX4, TLX5, and TLX 8 in the phase 2 of the decoys' screening in the SNI model. Triplexes contained I00 nmoles of each decoy and were dosed once at post-surgery day (POD) I4 and 23. (b) Effect of TLXI and of its individual decoys tested in parallel in the phase 3 of the screening. $t$ test versus vehicle, ${ }^{*} p<0.05, n=5-6$ per group. (c) Effect of TLX5 and of its individual decoys tested in parallel in the phase 3 of the screening, $t$ test versus vehicle, ${ }^{*} p<0.05, n=3$ per group. In all graphs, decoys' performance relating to the dual efficacy criteria are shown: von Frey values normed on PODI 4 preinjection values are displayed as white bars, and von Frey values normed on vehicle values at each tested day are displayed as black curves. Blue dashed lines mark the normed threshold value of I to assess decoys performance (black curves) against vehicle, red dashed lines mark the normed threshold value of 0.75 (i.e., $25 \%$ reduction) to assess decoys performance (white bars) against PODI4 preinjection von Frey values. von Frey values were normalized individually for each rat, and data are presented as means for each group; corresponding raw von Frey values are presented in supplemental Table 3. In (b) and (c) sections, TLXI and TLX5 contained 100 nmoles of each decoy, individual decoys were dosed at 200 nmoles and injections occurred once at day 14 and 17. Inclusion/exclusion based on predetermined criteria: two rats in (a) and three rats in (b) did not develop hypersensitivity following surgery, with PODI4 von Frey values $\leqslant 5$ and were excluded. The experiment in (c) was performed in two sequential cohorts of three animals per group with equal representation of testing conditions. All vehicle-treated animals of the first cohort showed a reduction of von Frey values in the 50\% range or below, with an average of $59 \%$ reduction between PODI4 and POD20 leading to the exclusion of that cohort (vehicle- plus decoy-treated animals). This study was performed at the Stanford University.

KLF: Kruppel-like transcription factor; POD: post-surgery day.

not pursued beyond day 31 . On the other end, KLF reference decoy 2 was not effective. When examining the sensitivity of the von Frey hairs affected by the efficacious decoys, the entire range was reduced from light $(1 \mathrm{~g})$ to strong mechanical stimuli $(26 \mathrm{~g})$ that elicit a paw withdrawal response even in the absence of an injury (Figure 4(f)). In the CCI model, the decoys also produced an array of efficacy patterns, $K L F$ decoys 1 and 2 being most efficacious (supplemental Table 4).

The combined analysis of decoy's KLF binding pattern and efficacy profiles in the SNI model highlights the following: (1) KLF9 binding appears to be a prerequisite to efficacy since $K L F$ reference decoy 2 is the only decoy to not bind KLF9 and to not show efficacy and 


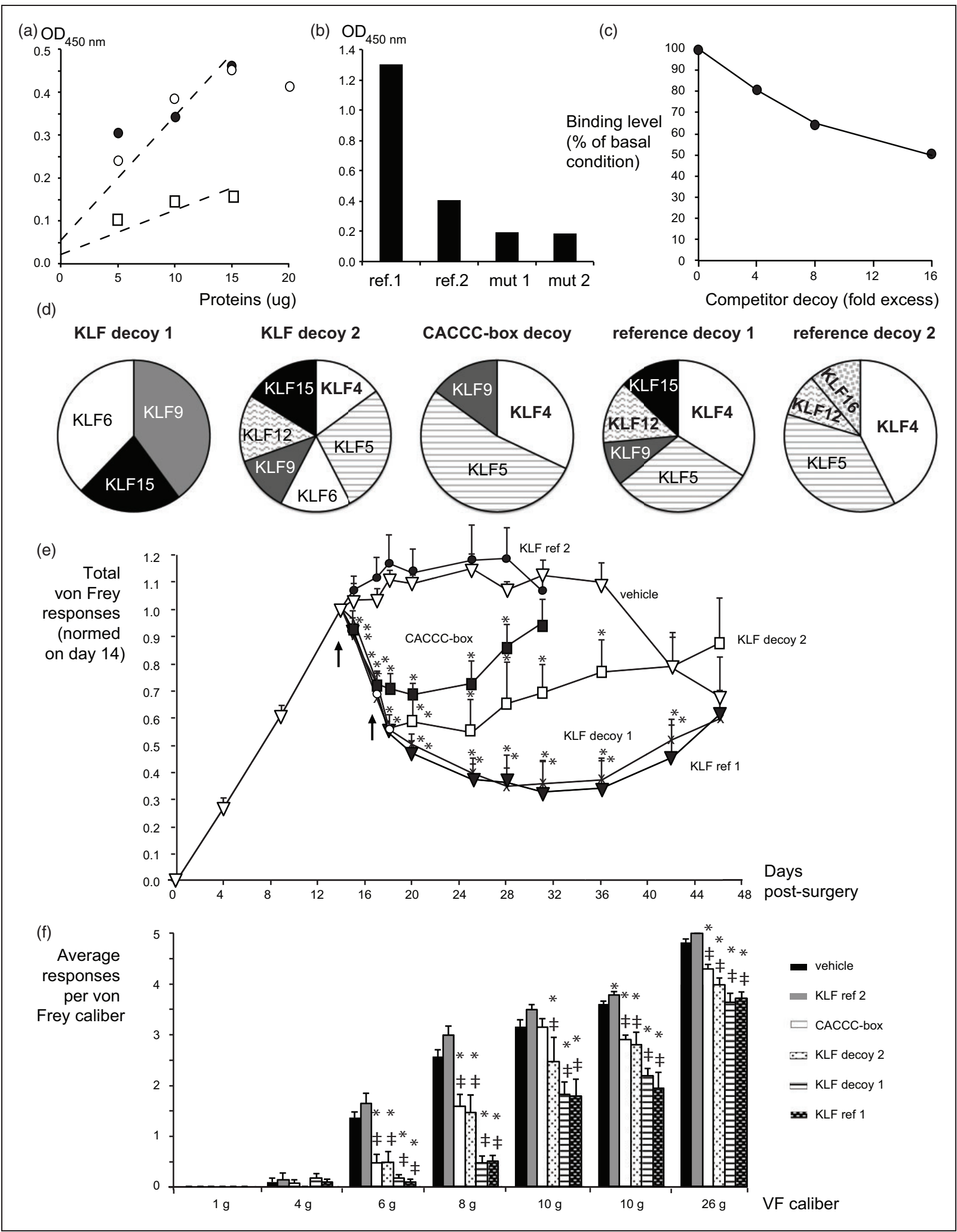

Figure 4. (a) KLF ELISA linearity and sensitivity. Increasing amounts of Hela nuclear protein extracts were incubated with a fixed amount of KLF decoy I biotinylated probe ( $12.5 \mu$ moles) and either I:1000 (square) or I:200 (black and white circles, two separate experiments) dilution of a specific KLF4 antibody. The signal produced by the $1: 200$ dilution plateaus after $15 \mu \mathrm{g}$ of protein. Regressions of the linear signal portion for each antibody dilution are shown as dotted line $\left(R^{2}=0.87\right.$ and $0.9 \mathrm{I}$ and $\mathrm{I}: 1000$ and $\mathrm{I}: 200$, respectively). (b) KLF ELISA specificity control I. The specificity of KLF binding is illustrated by the positive binding of reference decoys I and 2 to KLF4 and the lack of binding to KLF4 of two different mutant decoys lacking KLF binding sites. (c) KLF ELISA specificity control 2. Specificity of the assay was 
Table 2. Differential KLF binding of decoys.

\begin{tabular}{|c|c|c|c|c|c|c|c|c|c|c|c|c|c|c|c|c|c|c|}
\hline \multirow{3}{*}{$\begin{array}{l}\text { KLF } \\
\text { Member }\end{array}$} & \multirow{2}{*}{\multicolumn{3}{|c|}{$\frac{\mathrm{KLF}}{\text { Decoy I }}$}} & \multirow{2}{*}{\multicolumn{3}{|c|}{$\frac{\mathrm{KLF}}{\text { Decoy } 2}$}} & \multirow{2}{*}{\multicolumn{3}{|c|}{$\frac{\text { CACCC-box }}{\text { Decoy }}$}} & \multirow{2}{*}{\multicolumn{3}{|c|}{$\frac{\text { Reference }}{\text { Decoy I }}$}} & \multirow{2}{*}{\multicolumn{3}{|c|}{$\begin{array}{l}\text { Reference } \\
\text { Decoy } 2\end{array}$}} & \multirow{2}{*}{\multicolumn{3}{|c|}{$\frac{\text { Mutant }}{\text { Decoy I }}$}} \\
\hline & & & & & & & & & & & & & & & & & & \\
\hline & $M n$ & Md & SEM & $M n$ & Md & SEM & $M n$ & Md & SEM & $M n$ & Md & SEM & $M n$ & Md & SEM & $M n$ & Md & SEM \\
\hline KFL4 & 0.172 & 0.164 & 0.020 & 0.245 & 0.187 & 0.070 & 0.376 & 0.386 & 0.037 & 0.531 & 0.555 & 0.070 & 1.157 & 1.125 & 0.100 & 0.118 & 0.172 & 0.040 \\
\hline KFL5 & 0.306 & 0.292 & 0.030 & 0.452 & 0.466 & 0.050 & 0.622 & 0.496 & 0.100 & 0.477 & 0.503 & 0.100 & 1.009 & 0.990 & 0.080 & 0.307 & 0.275 & 0.050 \\
\hline KFL6 & 0.390 & 0.294 & 0.070 & 0.250 & 0.291 & 0.040 & 0.210 & 0.285 & 0.068 & 0.073 & 0.095 & 0.030 & 0.132 & 0.127 & 0.040 & 0.110 & 0.087 & 0.050 \\
\hline KFL9 & 0.216 & 0.214 & 0.030 & 0.195 & 0.184 & 0.030 & 0.176 & 0.153 & 0.040 & 0.148 & 0.089 & 0.040 & 0.206 & 0.045 & 0.140 & 0.002 & 0.000 & 0.000 \\
\hline $\mathrm{KFLI} 2$ & 0.337 & 0.317 & 0.030 & 0.235 & 0.236 & 0.020 & 0.258 & 0.252 & 0.021 & 0.210 & 0.220 & 0.010 & 0.251 & 0.254 & 0.010 & 0.297 & 0.276 & 0.030 \\
\hline KFLI5 & 0.369 & 0.372 & 0.010 & 0.266 & 0.257 & 0.040 & 0.087 & 0.028 & 0.066 & 0.205 & 0.208 & 0.010 & 0.177 & 0.177 & 0.010 & 0.185 & 0.184 & 0.000 \\
\hline KFLI 6 & 0.230 & 0.171 & 0.070 & 0.185 & 0.213 & 0.060 & 0.212 & 0.207 & 0.055 & 0.206 & 0.132 & 0.090 & 0.302 & 0.334 & 0.040 & 0.169 & 0.159 & 0.020 \\
\hline
\end{tabular}

Mean (Mn), median (Md), and SEM binding $\mathrm{OD}_{450}$ values (KLF ELISA) for each decoy to KLF4, KLF5, KLF6, KLF9, KLFI2, KLFI5, and KLFI6 are presented in the table. Student's $t$ test versus corresponding mutant decoy binding values: no highlight $=$ statistically significant $(p \leqslant 0.05)$, blue highlight $=$ statistical trend $(p \leqslant 0.1)$, gray highlight $=$ not statistically significant $(p>0.1)$. A hint of binding for KLF7 was detected for KLF decoy 2 and reference decoy 2; however, data could not be compared to the other KLF as it required using a recombinant version of the human KLF7 protein versus whole nuclear extracts for the other KLFs.

(2) KLF15 inhibition appears necessary for long-term efficacy and robust pain reduction as the $C A C C C$ box decoy, the active decoy with the lowest and shortest-lived efficacy, does not bind to KLF15 while the other active decoys do. Similar cross-analysis in the CCI model, in which KLF decoys 1 and 2 showed a meaningful efficacy and the CACCC-box decoy an intermediary efficacy level, suggests that (1) an appropriate level of inhibition of KLF9 is necessary but not sufficient for a robust efficacy since it is the common denominator between KLF decoys 1,2 and the CACCC-box decoy which present different efficacy levels (the fact that reference decoy 1 also inhibits KLF9 suggests that another factor it binds could interfere with its activity), and (2) KLF6 inhibition is required for a robust and sustained efficacy since only KLF decoys 1 and 2 have significant binding to KLF6. Those data suggest that KLF6, KLF9, and KLF15 cooperate to maintain chronic pain across the tested injuries, KLF9 and KLF15 in the SNI and KLF9 and KLF6 in the CCI models.

\section{AYX2 sequence development}

$K L F$ decoys 1 and 2 possess a unique ability to reduce pain by inhibiting KLF6, KLF9, and KLF15 activities. Those were selected as a basis to generate the sequence of a therapeutic decoy candidate for the treatment of chronic pain. The objectives for generating such sequence were to (1) reduce the sequence size below 25 bp (KLF decoy 1 and 2 are 37 and 29-bp long, respectively) to achieve appropriate solubility for clinical dosing (e.g., $110 \mathrm{mg} / \mathrm{mL}$ similar to AYX1 decoy, ClinicalTrials.gov identifier NCT02807428) and (2) maintain binding efficiency (i.e., binding activity

\footnotetext{
Figure 4. Continued

further demonstrated by the inhibition of KLF4 binding to the biotinylated KLF decoy I probe in the presence of increasing amount of free KLF decoy I competitor. This study was performed at Adynxx. (d) Decoys' KLF binding patterns. Pie graph representation of the proportionate binding of each specific KLF relative to the total binding to all bound KLFs for the corresponding decoy. Binding data significantly different from the binding level of mutant decoy I $(p \leqslant 0.05)$, or with a trend of statistical significance $(p \leqslant 0 . I)$, were used to generate the graphs (see Table 2), $n \geqslant 5$. This study was performed at Adynxx. (e) Effect of decoys with differential KLF binding patterns in the spared nerve injury (SNI) model. Mean + SEM values of normalized total responses to repetitive von Frey hair application for animals groups treated with vehicle (white triangle), KLF reference decoy I (black triangle), KLF reference decoy 2 (black circle), CACCC-box decoy (black square), KLF decoy I (X mark) and 2 (white square) are displayed. Normalization of von Frey hair responses was performed for each rat individually against its preinjection value measured on post-surgery day 14 , and the corresponding raw von Frey values are presented in supplemental Table 4. Articles were injected intrathecally once on day 14 and 17 (arrows). Testing of KLF decoys $I$ and 2 and reference decoy I, which presented the strongest activity level, was pursued until von Frey values started to resolve from a plateau level toward baseline in the vehicle group (day 48). Preinjection data before day 14 are combined across groups, 200 nmoles of each decoy or vehicle were injected once at days 14 and 17 (arrows), $t$ test versus vehicle: ${ }^{*} p \leqslant 0.05$, data distribution over the testing periods: ANOVA, all groups: $p<0.000$, Dunnett's multiple comparison test against vehicle: $p<0.000$ I for all groups except for $K L F$ ref $2(p=0.53), n=5$ per group. This study was performed at AfaSci, Inc. (f). Range of effect of the KLF decoys in the SNI model. Mean + SEM values of response for each individual von Frey hair out of five stimulations from the day of the first injection until the last day of testing for each decoy plotted from the experiment displayed in (e) above, ref $=$ reference, $t$ test versus vehicle, ${ }^{*} p<0.05$, ANOVA, for all groups at each hair level from 6 to $26 \mathrm{~g}: p<0.05$, Dunnett's multiple comparison test against vehicle: $\$ p<0.05$.

KLF: Kruppel-like transcription factor; VF: von Frey hair.
} 
Table 3. Binding profiles of KLF decoy $\mathrm{I}$ and 2 variants selected for in vivo testing.

\begin{tabular}{|c|c|c|c|c|c|c|c|c|}
\hline \multirow[b]{2}{*}{ Variant decoy name } & & & \multicolumn{3}{|c|}{ KLF decoy I variants } & \multicolumn{3}{|c|}{ KLF decoy 2 variants } \\
\hline & & & 1.6 .2 & 1.6 .5 & 1.9 & 2.1 & 2.5 & 2.9 \\
\hline \multicolumn{3}{|l|}{ Variant decoy size (bp) } & 25 & 18 & 22 & 15 & 24 & 21 \\
\hline \multicolumn{3}{|c|}{ Size reduction relative to original decoy (\%) } & $32 \%$ & $51 \%$ & $41 \%$ & $48 \%$ & $17 \%$ & $28 \%$ \\
\hline & \multicolumn{2}{|c|}{$\begin{array}{l}\text { Mean relative } \\
\text { binding efficiency }\end{array}$} & $148 \%$ & $127 \%$ & $134 \%$ & $168 \%$ & $141 \%$ & $128 \%$ \\
\hline Binding efficiency & KLF6 & Mean & $102 \%$ & $65 \%$ & $135 \%$ & $94 \%$ & $125 \%$ & $146 \%$ \\
\hline \multirow[t]{5}{*}{ relative to original decoy } & & SEM & $9 \%$ & $17 \%$ & $41 \%$ & $19 \%$ & $14 \%$ & $27 \%$ \\
\hline & KLF9 & Mean & $193 \%$ & $184 \%$ & $128 \%$ & $285 \%$ & $168 \%$ & $127 \%$ \\
\hline & & SEM & $10 \%$ & $40 \%$ & $8 \%$ & $116 \%$ & $14 \%$ & $15 \%$ \\
\hline & KLFI5 & Mean & $150 \%$ & $131 \%$ & $138 \%$ & $125 \%$ & $130 \%$ & $111 \%$ \\
\hline & & SEM & $5 \%$ & $10 \%$ & $16 \%$ & $25 \%$ & $5 \%$ & $7 \%$ \\
\hline
\end{tabular}

Relative binding efficiency is calculated as the binding of a decoy for a given KLF normalized by its sequence size and the binding efficiency of the decoy it derives from. Mean relative binding efficiency relates to the combined relative binding efficiency of KLF6, KLF9, and KLFI5; $n=4$ for each testing condition.

normalized by decoy size) to KLF6, KLF9, and KLF15 as decoy size decreases. The optimization process was conducted in two steps: (1) a first set of sequences was generated and tested to identify families of sequences with the potential to meet the targeted decoy features and then (2) a novel set of sequences was derived from the lead sequence families initially identified to further improve sequence features.

The binding of each decoy to KLF6, KLF9, and KLF15 was measured in duplicate and in parallel to $K L F$ decoys 1 and 2 using the KLF ELISA described above. When a sequence of interest was identified, its binding was confirmed independently once or twice more. Over 30 variant sequences were generated through this process by fusion, and orientation of the two KLF binding sites present in KLF decoys 1 and 2, as well as modifying the sequence and size of their 5' and 3' flanking sites. A variety of binding profiles were produced by those sequences, and six decoys $(1.6 .2,1.6 .5,1.9,2.1,2.5$, and 2.9) were selected as best matching the predetermined criteria (Table 3 ).

The effect of a single administration of each of these decoys was tested in parallel to KLF decoys 1 and 2 in the SNI and CCI models. Decoys or vehicle were injected on post-surgery day 14 , and their effects were measured up to day 31 , a time point sufficient to discriminate different efficacy patterns. Results highlighted a spectrum of decoy efficacy patterns in terms of amplitude and duration of pain relief across the two models (Figure 5(a)(c) and supplemental Table 5). Decoy 1.6.5 was selected as AYX2 because it has (1) the same efficacy as KLF decoy 1 in the SNI model and the highest combined efficacy across the SNI and CCI models (Figure 5(a)-(c)) and (2) a short size (18 bp) while maintaining the binding parameters of its parent KLF decoy 1 (37 bp; Figure 6(a)). The significant and long-lasting effects of a single administration of ascending doses of AYX2 were further confirmed in independent experiments in the SNI and CCI models (Figure 6(b)-(e)).

\section{Functional relationships associated with KLF6, KLF9,} and KLFI 5 binding

Binding to KLF9 appears central to a decoy efficacy, in association with KLF15 binding in the SNI model and with KLF6 binding in the CCI model. To understand the functional relationship between the bindings of each KLF pair, a detailed meta-analysis of in vivo and in vitro datasets generated during this body of work was conducted. It was hypothesized that a decoy efficacy could be driven by either the absolute binding level to its targets or by a ratio of binding to its targets. To test this, the average efficacy level of decoys in each pain model was analyzed as a potential linear function of KLF binding features. The analyses suggested that in the SNI model, the efficacy of a decoy is associated with its KLF15/KLF9 binding ratio (Figure 5(d)):

- If the binding level of KLF15 is similar to the binding level of KLF9 (i.e., KLF15/KLF9 0.9), the decoy has no effect.

- If the ratio of KLF15/KLF9 binding is unbalanced, a decoy efficacy increases as a linear function of the KLF15/KLF9 binding ratio (i.e., the more KLF15 binding over KLF9, the higher the efficacy, $\left.R^{2} \sim 0.7\right)$.

In the CCI model, the efficacy of a decoy appears in part correlated with its combined binding capacity to KLF6 + KLF9 (Figure 5(e)):

- The sole binding level to KLF6 (linear regression coefficient $\left.R^{2} \sim 0.3\right)$, KLF9 $\left(R^{2} \sim 0.4\right)$, or KLF15 $\left(R^{2} \sim 0.3\right)$ is not sufficient to explain efficacy. 
(a)

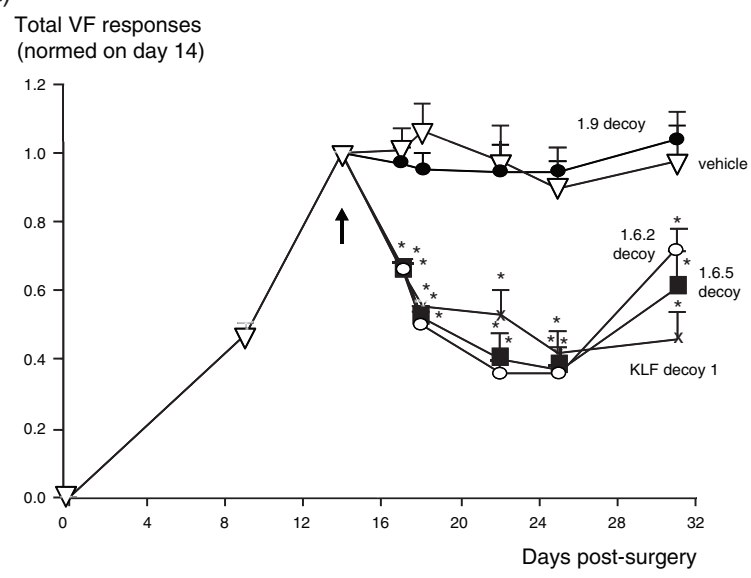

(c)

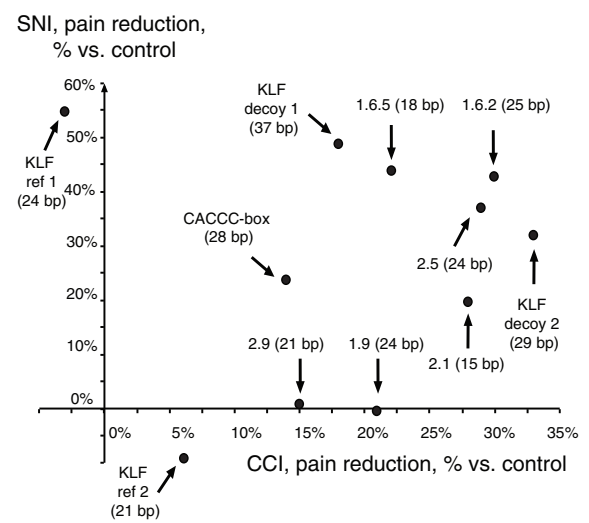

(d)

KLF15/KLF9 binding (b)
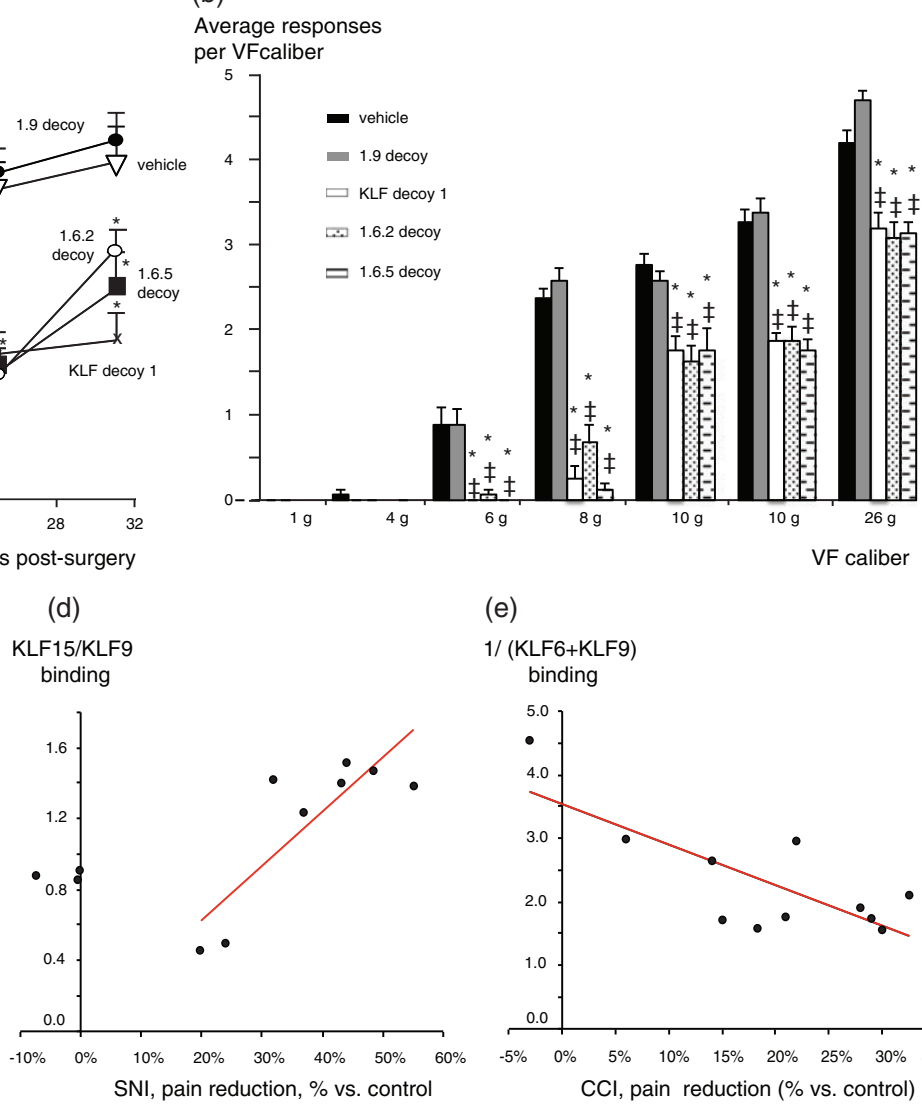

(e)

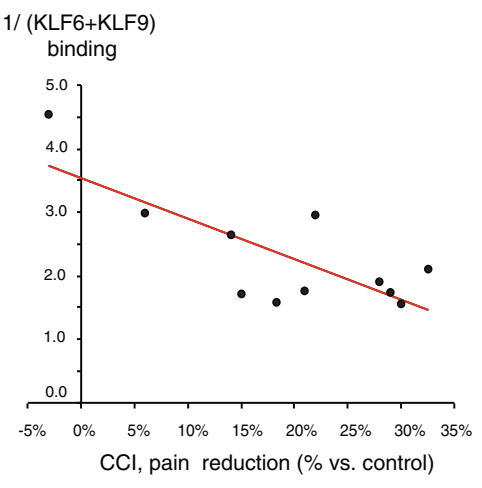

Figure 5. (a) Effect of variants of KLF decoy $I$ in the SNI model. Mean + SEM values of normalized total responses to repetitive von Frey hairs application for animal groups treated with vehicle (triangle), KLF decoy I (X mark), I.6.5 (white circle), I.6.2 (square), and I.9 (black circle) decoys are displayed. Normalization of von Frey hair responses was performed for each rat individually against its preinjection value measured on post-surgery day 14, and the corresponding raw von Frey values are presented in supplemental Table 5. Articles were injected intrathecally once on post-surgery day 14 (arrow); Preinjection data before day 14 are combined across groups, $t$ test versus vehicle: ${ }^{*} p \leqslant 0.05$, data distribution over the testing period: ANOVA, all groups: $p<0.000$ I, Dunnett's multiple comparisons test against vehicle: $p<0.05$ for all comparisons except for decoy 1.9, $n=4$ per group. This study was performed at AfaSci, Inc. (b) Range of effect of the variants of KLF decoy $I$ in the SNI model. Mean + SEM values of response for each individual von Frey hair out of five stimulations from the day of injection until the last day of testing for each decoy plotted from the experiment displayed in (a) above, ref $=$ reference, $t$ test versus vehicle, ${ }^{*} p<0.05$, ANOVA for all groups at each hair level from 6 to $26 \mathrm{~g}: p<0.05$, Dunnett's multiple comparison test against vehicle: $\$ p<0.05$. (c) Efficacy pattern of KLF decoys in the SNI and CCl pain models. Mean efficacy values for each decoy tested in the SNI and CCI models are plotted. Efficacy is calculated as the percentage of reduction of normalized von Frey hair response values compared to vehicle during the entire testing period (area under the curve) in each study and model, $n=4-6$ per decoy and model. (d) Linear regression of KLF decoy's efficacy in the SNI model in relation to KLFI5/KLF9 binding ratio. Efficacy is measured as described in (c) above. When a decoy efficacy was tested in independent studies, the value used for the plot is an average of the efficacy measured across those studies. Similarly, when a decoy binding was repeated throughout several phases of the sequence development process, the value used for the plot is an average of the binding measured across those phases. Each dot represents values of a given decoy, linear regression (red line), coefficient of linear regression $R^{2} \sim 0.7$. (e) Linear regression of KLF decoy efficacy in the CCl model in relation to KLF6+KLF9 binding. This plot was constructed using the principles described in (c). Each dot represents values of a given decoy, coefficient of linear regression $R^{2} \sim 0.6$. Studies included in (c), (d), and (e) were performed at the Stanford University and AfaSci, Inc.

$\mathrm{CCl}$ : chronic constriction injury; KLF: Kruppel-like transcription factor; SNI: spared nerve injury; VF: von Frey hair. 
Binding efficiency

(a) $\quad(x$ 0.001)

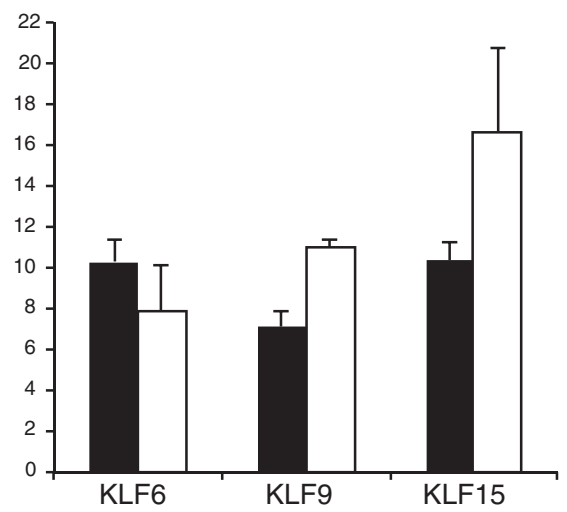

(b)

Total VF responses

(normed on day 14)

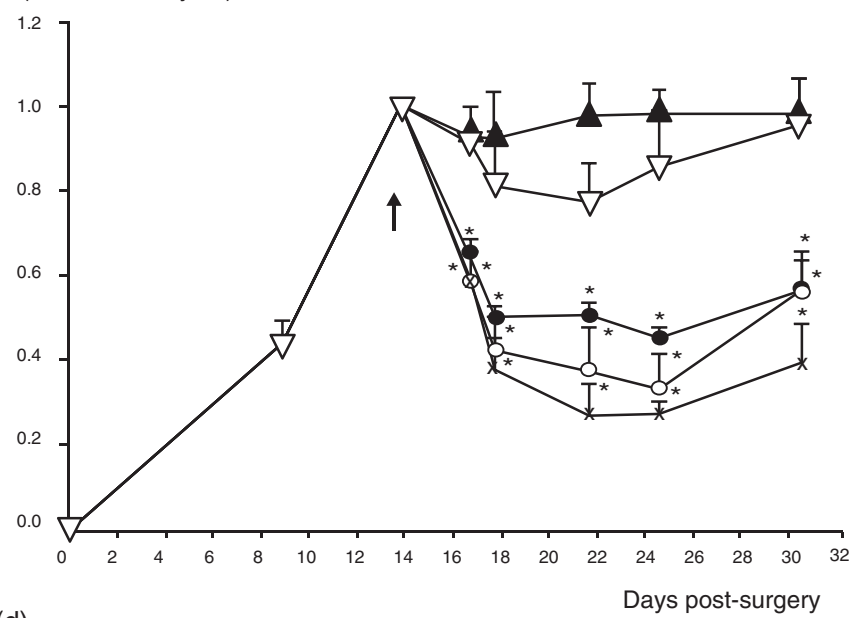

(d)

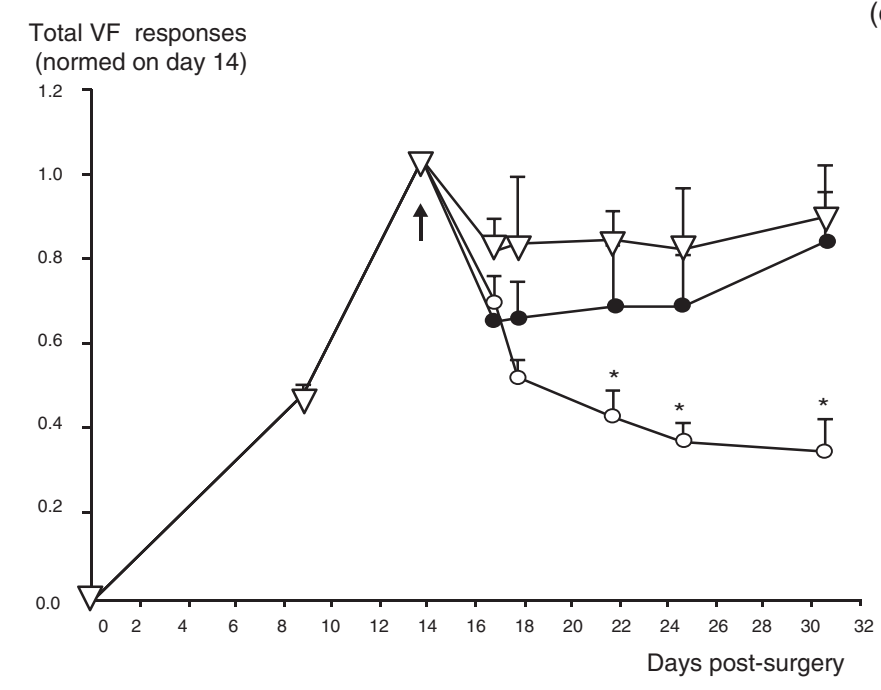

\begin{tabular}{|c|c|c|c|}
\hline Decoy & Size (bp) & $\begin{array}{c}\text { KLF15/KLF9 } \\
\text { ratio }\end{array}$ & $\begin{array}{c}\text { KLF6+KLF9 } \\
\text { binding } \\
\text { efficiency }\end{array}$ \\
\hline $\begin{array}{c}\text { KLF } \\
\text { decoy 1 }\end{array}$ & 37 & 1.46 & 0.017 \\
\hline AYX2 & 18 & 1.52 & 0.019 \\
\hline
\end{tabular}

(c)

Average responses per VFcaliber

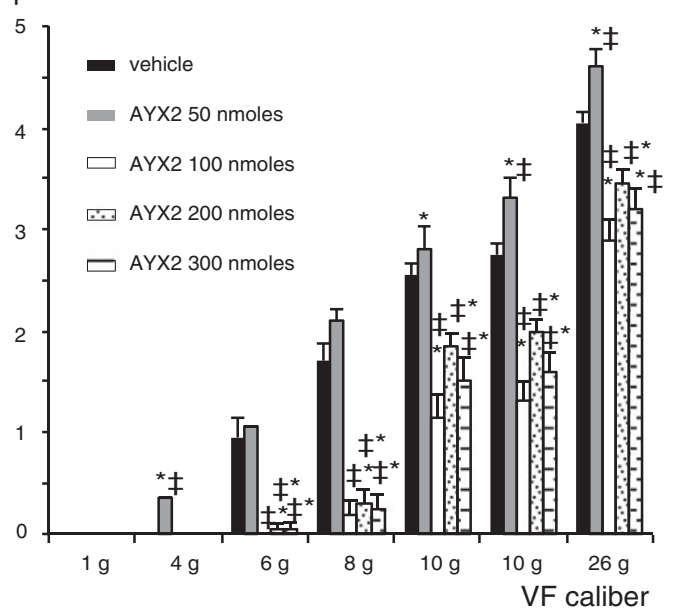

(e) Average responses per VF caliber

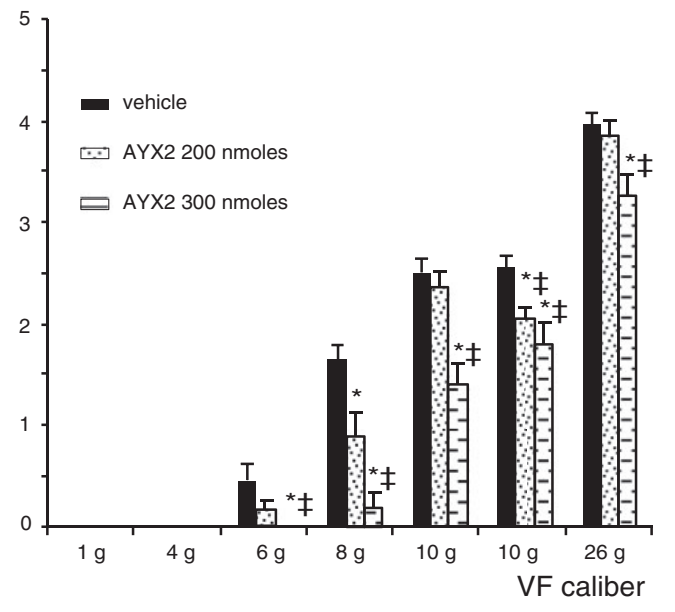

Figure 6. (a) Comparison of AYX2 (white bars) and KLF decoy I (black bars) binding features for KLF6, KLF9 and KLFI5. Mean + SEM binding efficiency for each KLF (binding level in $O D_{450}$ unit divided by the decoy size in bP) is shown in the graph; $n \geqslant 5$. This study was performed at Adynxx, Inc. (b) Effect of ascending AYX2 doses in the SNI model. Mean + SEM values of total responses to repetitive von Frey hair application for groups treated with vehicle (white triangle) or AYX2 50 (black triangle), 100 (X mark), 200 (black circle), or 300 nmoles (white circle) are displayed. In this experiment, the 50-nmole AYX2 dose is considered without effect based on the fact that von Frey responses remain stable following injection and do not increase nor decrease. Articles were injected intrathecally once on day 14 
- Combining KLF15 binding level to either KLF6 or KLF9 binding does not alter their respective linear regression coefficient (i.e., KLF6 $R^{2} \sim 0.3 \sim$ $\mathrm{KLF} 6+\mathrm{KLF} 15 R^{2} \sim 0.3$; KLF9 $R^{2} \sim 0.4 \sim \mathrm{KLF} 9+$ KLF15 $\left.R^{2} \sim 0.4\right)$, suggesting that KLF15 binding does not play a significant role in this type of pain.

- Efficacy appears to be a linear function of the combined binding of KLF6 + KLF9 ( $R^{2}$ of $1 /$ (KLF6 + KLF9) $\sim 0.6$ ): the more the binding, the higher the efficacy. Considering the value level of the regression coefficient, it is conceivable that other factors potentially independent of binding activities, such as stability or cellular uptake influence a decoy's activity. Collectively, this meta-analysis shows that the relationships between KLF9 and KLF15 or KLF9 and KLF6 are different and work independent of each other.

\section{Discussion}

\section{Effects of intrathecal AYX2 and binding to KLF6, KLF9,} and KLFI5

Pain is a dynamic state maintained at the genomic level by dynamic transcriptomes. ${ }^{1,28}$ A group of transcription factors with the potential to control these transcriptomes was selected based on their known functions and gene promoter's analysis. The impartial screening of decoys designed against those factors identified two decoys that bind to KLF6, KLF9, and KLF15 that significantly reduced chronic pain following a single administration in the SNI and CCI models of pain.

Based on prior work using repeat von Frey testing, ${ }^{18,25}$ the magnitude of von Frey responses at the time of the decoy treatments reflected a fully developed mechanical hypersensitization and nociceptive state. In that context, KLF decoys produced up to $\sim 60 \%-70 \%$ reduction in mechanical hypersensitivity compared to controls, and the effect lasted until hypersensitivity was resolving in controls. Based on the correlation between ELISA binding data and in vivo efficacy levels analyses,
KLF9 binding appears to be the central component of the trio of KLFs bound by the decoys, in association with KLF15 binding to treat pain in the SNI model and with KLF6 binding in the CCI model. The functional relationships between KLF9 binding and binding to the two other KLFs are not of similar nature, suggesting that different transcriptional mechanics underlie pain maintenance in the two pain models. This observation is consistent with head-to-head microarray comparison of spinal cord gene regulations showing that the amount and type of genes regulated are principally different in the SNI and CCI models. ${ }^{29}$

AYX2 was developed as a KLF decoy drug candidate with a short size capable to achieve a solubility level compatible with clinical use. Prior pharmacodynamic, pharmacokinetic, and metabolism studies in rats with AYX1 showed that a short decoy, unprotected from metabolism, acts locally in the DRG and/or the spinal cord tissues following an intrathecal administration. ${ }^{18,25,30}$ Further, this work showed that AYX1 rapidly penetrates the DRG and spinal cord following administration and that cell uptake likely occurs in a nonspecific fashion in both neurons and glial cells via endocytic mechanisms. ${ }^{23}$ Considering the doses used with AYX2 are similar to AYX1, that both decoys are of comparable size and unprotected toward metabolism, an analogous local tissue and cells distribution is anticipated. Independent work in mice shows that KLF6, KLF9, and/or KLF15 are expressed in the DRG and/ or the spinal cord in basal or pathologic nociceptive conditions. ${ }^{31-33}$ Determining whether AYX2 site of action is primarily the DRG, the spinal cord or both in the SNI and CCI models would constitute a next step in the characterization of AYX2 mechanism of action.

\section{Potential roles of KLF6, KLF9, and KLFI5 in nociception}

Uncovering the potential functions of KLF6, KLF9, and KLF15 in the DRG and/or spinal cord in relation to

Figure 6. Continued

(arrow), $t$ test versus vehicle: ${ }^{*} p \leqslant 0.05$, data distribution over the testing period: ANOVA, all groups: $p<0.000$ I, Dunnett's multiple comparisons test against vehicle: $p<0.05$ for all comparisons, $n=4$ per group. (c) Range of effect of increasing dose levels of AYX2 in the $\mathrm{SNI}$ model. Mean + SEM values of response for each individual von Frey hair out of five stimulations over the testing period from the day of injection until the last day of testing plotted from the experiment displayed in (b) above, $t$ test versus vehicle, $* p<0.05$, ANOVA for all groups at each hair level from 4 to $26 \mathrm{~g}$ : $p<0.0005$, Dunnett's multiple comparison test: $\$ p<0.05$. (d) Effect of ascending AYX2 dose levels in the $\mathrm{CCl}$ model. Mean + SEM values of total responses to repetitive von Frey testing for animals groups treated with vehicle (white triangle) or AYX2 200 (black circle) or 300 nmoles (white circle) are displayed. Articles were injected intrathecally once on day I4 (arrow), $t$ test versus vehicle: $* p \leqslant 0.05$, data distribution over the testing period: ANOVA, all groups: $p<0.000$, Dunnett's multiple comparisons test against vehicle: $p<0.05$ for all comparisons, $n=4$ per group. (e) Range of effect of increasing dose levels of $A Y X 2$ in the $C C l$ model. Mean + SEM values of response for each individual von Frey hair out of 5 stimulations over the testing period from the day of injection until the last day of testing plotted from the experiment displayed in (d) above, $t$ test versus vehicle, $* p<0.05$, ANOVA, for all groups at each hair level from 6 to $26 \mathrm{~g}: p<0.05$, Dunnett's multiple comparison test against vehicle: $\$ p<0.05$. Studies presented parts (b)-(e) were performed at AfaSci, Inc.

KLF: Kruppel-like transcription factor; VF: von Frey hair. 
nociception requires experimental determination and would be a direct expansion of the work presented here. However, hypotheses can be drawn based on the known roles of KLF6, KLF9, and KLF15 in the central nervous system, and we propose in the section below mechanisms that could be at play in relation to AYX2 efficacy.

The nociceptive system functions through long-term potentiation and memory mechanisms similar to the hippocampus. ${ }^{34}$ Studies of the hippocampus in KLF9 knockout versus wild-type mice showed that KLF9 is expressed in neurons and required for neurogenesisdependent synaptogenesis in adults. ${ }^{35}$ Further, KLF9 expression is induced by neuronal activity and regulates genes involved in synaptic plasticity (e.g., Lin7b, pentraxin-2, Nav1.3, $\mathrm{Ca}_{\mathrm{v}} 3.1$, KChip4, KCNA4). ${ }^{36-42}$ It is possible that KLF9 controls the transcriptomes maintaining synaptic structural rearrangement and hyperexcitability following injury in the DRG and spinal cord, two landmark mechanisms of chronic pain. While KLF15 can be expressed in neurons, KLF15 is sufficient to induce an astrocyte phenotype and appears to be mainly expressed in GFAP-positive astrocytes in the adult spinal cord. ${ }^{32}$ The fact that AYX2 efficacy is concurrent with KLF9 and KLF15 binding at a ratio different from $\sim 1$ in the SNI model suggests that the physiological output of KLF9 and KLF15 functions as a ratio of their respective activities. Similar mechanisms have been illustrated in endocrine systems, where KLF9 and KLF15 are implicated in feed forward loops and synergistic transcriptional mechanisms. ${ }^{43}$ Thus an appropriate ratio of transcriptional activity of KLF9 in neurons and KLF15 in astrocytes may be required in the DRG-spinal cord network for the two cell types to efficiently interact and coordinate the perpetuation of chronic pain. This may be achieved by locally maintaining high activity levels of bi- (pre- and post-synaptic neurons) and tri- (including astrocytes) partite synapses. Such intercellular cross talk would be consistent with KLF physiology, as illustrated by KLF4 and KLF5 which coordinate epithelial cell proliferation while being expressed in different cell types. ${ }^{26}$ In such context, an hypothesis is that AYX2 treatment could restore the ratio of KLF15 and KLF9 activities in neurons and astrocytes to a baseline level, disrupting the pathologic state of communication between those cells required for high synaptic activity and chronic pain.

In the CCI model, our data suggest that AYX2 activity is driven by total binding to KLF6 plus KLF9, independent of the ratio of binding, which would indicate that KLF6 and KLF9 roles are redundant for maintaining chronic pain. Similar functional redundancy has been demonstrated between KLF6 and KLF7 which can compensate for each other to drive neurite outgrowth. ${ }^{44}$ Further, multiple KLFs can act as a network to regulate common intracellular pathways. ${ }^{43}$ Since KLF9 is involved in synaptogenesis, one can envision KLF6 and KLF9 both maintaining the synaptic changes required for neuronal sensitization and pain in the DRG-spinal cord network. Interestingly, a side-by-side electrophysiological study of the DRG-spinal cord connectivity in the SNI and CCI models revealed "divergent plasticity" mechanisms of excitatory synaptic transmission in the two models. ${ }^{45}$ This data is consistent with KLF9 function in synaptic plasticity and suggests that KLF9 and KLF15 on one hand and KLF9 and KLF6 on the other hand build distinct modalities of plasticity in the two models.

\section{KLF6, KLF9, and KLFI5 in rodents and human}

KLF6, KLF9, and KLF15 are well-conserved ortholog proteins with $94 \%, 97 \%$, and $84 \%$ protein identity, respectively, between rat and human forms. Further, an almost complete conservation is found within their zinc finger DNA binding sites with sequence identities ranging from $97 \%$ to $100 \%$ across the two species (NCBI accession numbers: NP_113830/NP_001291, NP_476559/NP_001197, AAH89782/NP_054798).

This structural conservation appears to correlate, at least in the systems that have been studied below, to a conservation of function between rodents and human. For instance, experiments in mice and in human endothelial cells showed that KLF6 promotes vascular remodeling following an injury in both species. ${ }^{46} \mathrm{Head}-$ to-head study in mice and human primary hepatocytes showed that KLF9 was induced by the triiodothyronine hormone and controlled the downstream hepatocytes proliferation and differentiation response. ${ }^{47}$ As for KLF15, it is upregulated by glucocorticoids in human and mouse airway smooth muscles and acts as a regulator of airway function, illustrating conservation of both function and regulation. ${ }^{48,49}$ This combined information on the conservation of KLF structure, regulation, and function supports the potential translation of AYX2 drug candidate effects from rats to human and highlights KLF6, KLF9, and KLF15 as novel targets for the treatment of pain.

\section{Conclusions}

This study shows that KLF decoys binding to KLF6, KLF9, and KLF15 transcription factors can alleviate mechanical hypersensitivity in the SNI and CCI rat models of chronic neuropathic pain. The correlation of in vitro decoy binding data with in vivo efficacy suggests KLF9 cooperates in different fashions with KLF6 and KLF15 depending on the pain etiology, which is consistent with the modus operandi of this large family of integrated transcription factors. AYX2 is an 18-bp long DNA decoy developed specifically to bind and inhibit 
KLF6, KLF9, and KLF15 locally in the DRG spinal cord network to produce large and long-lasting relief of mechanical hypersensitivity following a single intrathecal injection. This data support AYX2 therapeutic potential for treating chronic pain syndromes and constitutes the basis for characterizing further the roles of the KLF family in nociception.

\section{Acknowledgments}

The authors thank Drs Tony Yaksh (University of California San Diego), William K. Schmidt (NorthStar Consulting, LLC), George Miljanich (In memoriam, SiteOne Therapeutics, Inc.), Rick Orr, William Martin, Kim Herbert, and Dina Gonzalez from Adynxx, Inc. for their great support and guidance in the accomplishment of this work.

\section{Author Contributions}

Julien Mamet, Donald Manning, and Scott Harris designed and monitored this work. Michael Klukinov, David Yeomans, Conrado Pascual, and Simon Xie performed pharmacology experiments. Julien Mamet also designed the decoys described in the study and performed the ELISA experiments. Brad Taylor and Renee Donahue guided pharmacology study designs. All authors read and approved the final manuscript.

\section{Declaration of Conflicting Interests}

The author(s) declared the following potential conflicts of interest with respect to the research, authorship, and/or publication of this article: Julien Mamet, Donald Manning, and Scott Harris are Adynxx employees and shareholders. David Yeomans and Brad Taylor are Adynxx consultants and/or shareholders.

\section{Funding}

The author(s) disclosed receipt of the following financial support for the research, authorship, and/or publication of this article: This study was funded by Adynxx, Inc.

\section{References}

1. Lacroix-Fralish ML, Tawfik VL, Tanga FY, et al. Differential spinal cord gene expression in rodent models of radicular and neuropathic pain. Anesthesiology 2006; 104: 1283-1292.

2. Xiao HS, Huang QH, Zhang FX, et al. Identification of gene expression profile of dorsal root ganglion in the rat peripheral axotomy model of neuropathic pain. Proc Natl Acad Sci U S A 2002; 99: 8360-8365.

3. Yukhananov R and Kissin I. Persistent changes in spinal cord gene expression after recovery from inflammatory hyperalgesia: a preliminary study on pain memory. $B M C$ Neurosci 2008; 9: 32.

4. Aley KO, Martin A, McMahon T, et al. Nociceptor sensitization by extracellular signal-regulated kinases. J Neurosci 2001; 21: 6933-6939.
5. Caterina MJ, Rosen TA, Tominaga M, et al. A capsaicinreceptor homologue with a high threshold for noxious heat. Nature 1999; 398: 436-441.

6. Cockayne DA, Hamilton SG, Zhu QM, et al. Urinary bladder hyporeflexia and reduced pain-related behaviour in P2X3-deficient mice. Nature 2000; 407: 1011-1015.

7. Dowd E, McQueen DS, Chessell IP, et al. P2X receptormediated excitation of nociceptive afferents in the normal and arthritic rat knee joint. Br J Pharmacol 1998; 125: 341-346.

8. Kerr BJ, Souslova V, McMahon SB, et al. A role for the TTX-resistant sodium channel Nav 1.8 in NGF-induced hyperalgesia, but not neuropathic pain. Neuroreport 2001; 12: $3077-3080$.

9. Obata $\mathrm{K}$ and Noguchi K. MAPK activation in nociceptive neurons and pain hypersensitivity. Life Sci 2004; 74: 2643-2653.

10. Ossipov MH, Bazov I, Gardell LR, et al. Control of chronic pain by the ubiquitin proteasome system in the spinal cord. J Neurosci 2007; 27: 8226-8237.

11. Song XJ, Wang ZB, Gan Q, et al. cAMP and cGMP contribute to sensory neuron hyperexcitability and hyperalgesia in rats with dorsal root ganglia compression. J Neurophysiol 2006; 95: 479-492.

12. Suzuki R and Dickenson A. Spinal and supraspinal contributions to central sensitization in peripheral neuropathy. Neurosignals 2005; 14: 175-181.

13. Waldmann R, Champigny G, Bassilana F, et al. A protongated cation channel involved in acid-sensing. Nature 1997; 386: 173-177.

14. Wang H and Woolf CJ. Pain TRPs. Neuron 2005; 46: 9-12.

15. Woolf CJ. Phenotypic modification of primary sensory neurons: the role of nerve growth factor in the production of persistent pain. Philos Trans R Soc Lond B Biol Sci 1996; 351: 441-448.

16. Jones MW, Errington ML, French PJ, et al. A requirement for the immediate early gene Zif268 in the expression of late LTP and long-term memories. Nat Neurosci 2001; 4: 289-296.

17. Ko SW, Vadakkan KI, Ao H, et al. Selective contribution of Egrl (zif/268) to persistent inflammatory pain. J Pain 2005; 6: 12-20.

18. Mamet J, Klukinov M, Yaksh TL, et al. Single intrathecal administration of the transcription factor decoy AYX1 prevents acute and chronic pain after incisional, inflammatory, or neuropathic injury. Pain 2014; 155: 322-333.

19. Matsumoto N, Laub F, Aldabe R, et al. Cloning the cDNA for a new human zinc finger protein defines a group of closely related Kruppel-like transcription factors. J Biol Chem 1998; 273: 28229-28237.

20. Shields JM and Yang VW. Identification of the DNA sequence that interacts with the gut-enriched Kruppellike factor. Nucleic Acids Res 1998; 26: 796-802.

21. Heyman John CB, Wasden Chris and Baer Sarah. Quantitation of activated transcription factors for drug discovery using TransAM ${ }^{\mathrm{TM}}$ ELISA kits. Nat Methods2006. doi:10.1038/an1451.

22. Bennett GJ and Xie YK. A peripheral mononeuropathy in rat that produces disorders of pain sensation like those seen in man. Pain 1988; 33: 87-107. 
23. Butler SH, Godefroy F, Besson JM, et al. A limited arthritic model for chronic pain studies in the rat. Pain 1992; 48: 73-81.

24. Decosterd I and Woolf CJ. Spared nerve injury: an animal model of persistent peripheral neuropathic pain. Pain 2000; 87: 149-158.

25. Mamet J, Harris S, Klukinov M, et al. Pharmacology, pharmacokinetics, and metabolism of the DNA-decoy AYX1 for the prevention of acute and chronic post-surgical pain. Mol Pain 2017; 13: 1744806917703112.

26. Dang DT, Zhao W, Mahatan CS, et al. Opposing effects of Kruppel-like factor 4 (gut-enriched Kruppel-like factor) and Kruppel-like factor 5 (intestinal-enriched Kruppellike factor) on the promoter of the Kruppel-like factor 4 gene. Nucleic Acids Res 2002; 30: 2736-2741.

27. McConnell BB and Yang VW. Mammalian Kruppel-like factors in health and diseases. Physiol Rev 2010; 90: 1337-1381.

28. LaCroix-Fralish ML, Austin JS, Zheng FY, et al. Patterns of pain: meta-analysis of microarray studies of pain. Pain 2011; 152: 1888-1898.

29. Griffin RS, Costigan M, Brenner GJ, et al. Complement induction in spinal cord microglia results in anaphylatoxin C5a-mediated pain hypersensitivity. J Neurosci 2007; 27: 8699-8708.

30. Mamet J, Yeomans DC, Yaksh T, et al. Formulation and toxicology evaluation of the intrathecal AYX1 DNAdecoy in Sprague Dawley rats. Toxicol Sci. Epub ahead of print 19 June 2017. DOI: https://doi.org/10.1093/ toxsci/kfx118.

31. Allen Brain Atlas, http://mousespinal.brain-map.org/, experiments 100007104, 100024380, 100017398, $100017397,100033071$.

32. Fu H, Cai J, Clevers H, et al. A genome-wide screen for spatially restricted expression patterns identifies transcription factors that regulate glial development. J Neurosci 2009; 29: 11399-11408.

33. Nilsson A, Moller K, Dahlin L, et al. Early changes in gene expression in the dorsal root ganglia after transection of the sciatic nerve; effects of amphiregulin and PAI1 on regeneration. Brain Res Mol Brain Res 2005; 136: 65-74.

34. Sandkuhler J. Learning and memory in pain pathways. Pain 2000; 88: 113-118.

35. Scobie KN, Hall BJ, Wilke SA, et al. Kruppel-like factor 9 is necessary for late-phase neuronal maturation in the developing dentate gyrus and during adult hippocampal neurogenesis. J Neurosci 2009; 29: 9875-9887.
36. Huguenard JR. Low-threshold calcium currents in central nervous system neurons. Ann Rev Physiol 1996; 58: 329-348.

37. Lujan R, de Cabo de la Vega C, Dominguez del Toro E, et al. Immunohistochemical localization of the voltagegated potassium channel subunit Kv1.4 in the central nervous system of the adult rat. J Chem Neuroanat 2003; 26: 209-224.

38. Olsen O, Moore KA, Nicoll RA, et al. Synaptic transmission regulated by a presynaptic MALS/Liprin-alpha protein complex. Curr Opin Cell Biol 2006; 18: 223-227.

39. Rhodes KJ, Carroll KI, Sung MA, et al. KChIPs and Kv4 alpha subunits as integral components of A-type potassium channels in mammalian brain. $J$ Neurosci 2004; 24: 7903-7915.

40. Schmidt-Hieber C, Jonas P and Bischofberger J. Enhanced synaptic plasticity in newly generated granule cells of the adult hippocampus. Nature 2004; 429: 184-187.

41. Xu D, Hopf C, Reddy R, et al. Narp and NP1 form heterocomplexes that function in developmental and activitydependent synaptic plasticity. Neuron 2003; 39: 513-528.

42. Yu FH and Catterall WA. Overview of the voltage-gated sodium channel family. Genome Biol 2003; 4: 207.

43. Knoedler JR and Denver RJ. Kruppel-like factors are effectors of nuclear receptor signaling. Gen Comp Endocrinol 2014; 203: 49-59.

44. Moore DL, Apara A and Goldberg JL. Kruppel-like transcription factors in the nervous system: novel players in neurite outgrowth and axon regeneration. Mol Cell Neurosci 2011; 47: 233-243.

45. Kohno T, Moore KA, Baba $\mathrm{H}$, et al. Peripheral nerve injury alters excitatory synaptic transmission in lamina II of the rat dorsal horn. $J$ Physiol 2003; 548: 131-138.

46. Gallardo-Vara E, Blanco FJ, Roque $M$, et al. Transcription factor KLF6 upregulates expression of metalloprotease MMP14 and subsequent release of soluble endoglin during vascular injury. Angiogenesis 2016; 19: 155-171.

47. Cvoro A, Devito L, Milton FA, et al. A thyroid hormone receptor/KLF9 axis in human hepatocytes and pluripotent stem cells. Stem Cells 2015; 33: 416-428.

48. Masuno K, Haldar SM, Jeyaraj D, et al. Expression profiling identifies Klf15 as a glucocorticoid target that regulates airway hyperresponsiveness. Am J Respir Cell Mol Biol 2011; 45: 642-649.

49. Sasse SK, Kadiyala V, Danhorn T, et al. Glucocorticoid receptor ChIP-seq identifies PLCD1 as a KLF15 target that represses airway smooth muscle hypertrophy. Am J Respir Cell Mol Biol 2017; 57: 226-237. 